Ariel T. Levy ${ }^{1}$ / Melissa A. Yurkanin² / Lauren A. Plante ${ }^{2}$

\title{
Fetal head entrapment within the lower uterine segment
}

\author{
${ }^{1}$ Department of Obstetrics and Gynecology, Hahnemann University Hospital, 245 N. $15^{\text {th }}$ Street MS 495, Philadelphia, PA 19192 , \\ USA, Tel.: 484-574-6037, E-mail: ariels5108@gmail.com. https://orcid.org/0000-0002-5348-9300. \\ ${ }^{2}$ Department of Obstetrics and Gynecology, Hahnemann University Hospital, Philadelphia, PA, USA
}

\begin{abstract}
:
Fetal head entrapment by a uterine scar or adhesion is a rare obstetric complication. We present a case of a uterine constriction diagnosed in pregnancy that resulted in confinement of the fetal head to the lower uterine segment. The developing fetus ultimately suffered growth restriction of the head and was delivered after the mother experienced preterm premature rupture of membranes (PROM). Severe adhesions of the lower uterine segment were confirmed during the patient's classical cesarean section.
\end{abstract}

Keywords: Classical cesarean delivery, fetal head entrapment, growth restriction, prenatal ultrasonography, uterine adhesions

DOI: 10.1515/crpm-2019-0043

Received: June 16, 2019; Accepted: September 5, 2019

\section{Introduction}

Adhesive disease involving the lower uterine segment is a common complication of cesarean sections. Prior studies have found increased adverse pregnancy outcomes and fetal morbidity in subsequent pregnancies [1]. Fortunately, fetal head entrapment by a uterine adhesion is a rare occurrence. This article presents a case of a uterine constriction diagnosed on ultrasound that resulted in fetal head entrapment within the lower uterine segment and the subsequent complications.

\section{Case presentation}

A 34-year-old woman (G6P2032) was first found to have a thickened, inward projecting lower uterine segment during her fetal anatomy ultrasound at $22+1 / 7$ weeks' gestation. This fibrous band appeared to be circumferentially encompassing the fetal neck and restricting the movement of the fetal head. This was considered to possibly represent a uterine band, amniotic band or sequela of a previous cesarean delivery. The patient's pregnancy had been uncomplicated up until this point. A 13-week ultrasound had not demonstrated this finding.

The patient had a history of two prior full-term cesarean deliveries. The first, 14 years earlier, had been complicated by the formation of a uterine hematoma along the superior aspect of the hysterotomy, which was decompressed with several figure-of-eight stitches. Significant intra-abdominal adhesions and a large uterine window were noted during her second cesarean delivery 2 years later. The patient otherwise had several elective abortions that were managed surgically with dilation and evacuation. The remainder of the patient's medical history was unremarkable except for a body mass index of 35.

At a follow up ultrasound at $26^{+1 / 7}$ weeks, the fetal growth was appropriate for gestational age and the amniotic fluid index was normal, but the fetal neck remained constricted beneath the uterine band (Figure 1). The recommendation was made for a follow-up magnetic resonance imaging (MRI) to better characterize the pathology of the lower uterine segment, but at 29 weeks, before the MRI had been performed, the patient presented to labor and delivery with preterm premature rupture of membranes (PPROM). She was hospitalized and managed in the usual way, with antenatal corticosteroids and latency antibiotics. Twice-daily non-stress tests were reactive during her hospital course. 


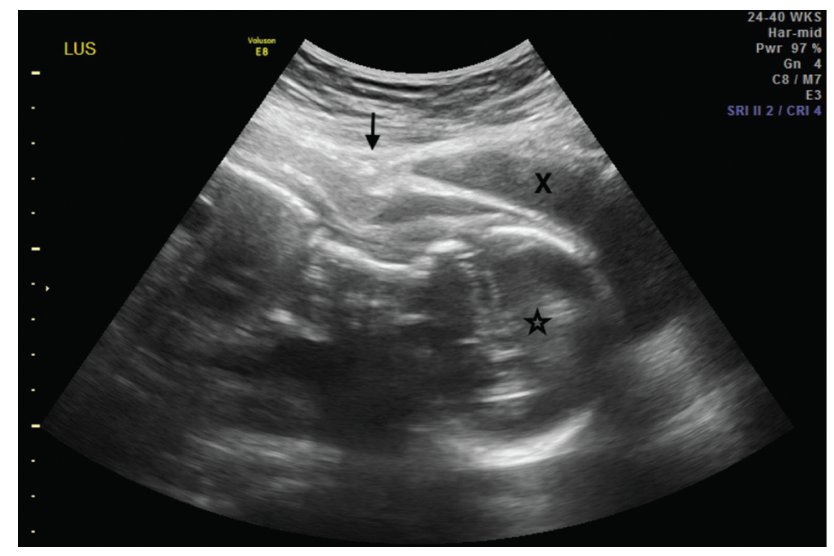

Figure 1: Sagittal sonographic view of lower uterine segment showing an adhesive band (arrow) at the level of the fetal neck confining the fetal head (star) to the lower uterine segment $(X)$.

At $30^{+1 / 7}$ weeks' gestation, the patient had a repeat growth scan performed that noted an unchanged lower uterine segment. The estimated fetal weight of $1337 \mathrm{~g}$ was overall appropriate for gestational age, but the head circumference was less than the second percentile by Hadlock growth curves. Umbilical artery Dopplers were normal. A single 1-cm pocket of amniotic fluid was appreciated, consistent with the rupture of membranes. No fetal movement or breathing was appreciated during the exam, and the biophysical profile (BPP) score was 0 out of 8 . We speculated that external constraints on the fetus might limit fetal movement and that the BPP might therefore be falsely low, rather than reflective of fetal compromise. Given the physical constraints of the intrauterine environment and the previously reassuring non-stress tests, the plan was made for the patient to undergo continuous fetal monitoring to assess for acute fetal compromise. Over the next $24 \mathrm{~h}$, there was an isolated late deceleration, but was otherwise category 1 with moderate variability and accelerations. The patient was counseled on cesarean delivery secondary to a category 2 tracing, signs of restricted growth of the fetal head, and concern about prolonged fetal immobility. She received magnesium for neuroprotection and proceeded to delivery.

A Pfannenstiel skin incision was made and upon entry into the abdomen, dense adhesions were noted between the uterus and rectus muscles, extending from the mid uterus down to the underdeveloped lower uterine segment. A band of adhesive tissue encased the bladder and entirely obliterated the vesicouterine space (Figure 2). Due to the inability to safely reach the lower uterine segment, a decision was made to proceed with a classical uterine incision and breech extraction. The fetal body was delivered without difficulty, but the tight band around the neck made delivery of the fetal head more challenging. The surgeon was able to squeeze a hand beneath the band and deliver the fetus. After delivery of the infant, careful dissection of the fascia from the underlying tissue was done. The scarred lower uterine segment was adherent to the anterior abdominal wall, but was eventually visualized after extensive lysis of adhesions (Figure 3). An intraoperative urology consult ensured there was no evidence of bladder injury. The infant's Apgar scores were 6, 7, and 8 at 1, 5, and $10 \mathrm{~min}$, respectively. Initial umbilical artery cord gas noted a pH of 7.281 with base excess of 2.1. The infant's head on the first day of life was $28 \mathrm{~cm}$, consistent with measurements on the ultrasound. On postoperative day 4, the patient was discharged home, but the infant remained in the neonatal intensive care unit (NICU) for approximately 6 weeks after delivery.

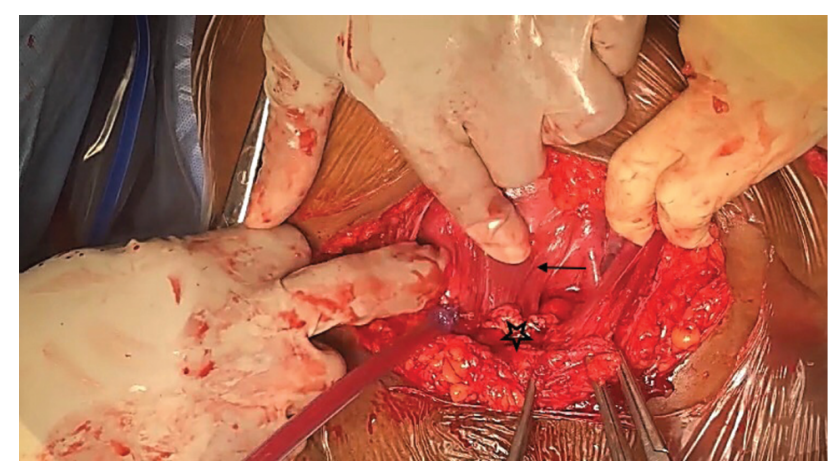

Figure 2: Adhesive disease between uterus (arrow) and anterior abdominal wall (star) obliterating the vesicouterine space. 


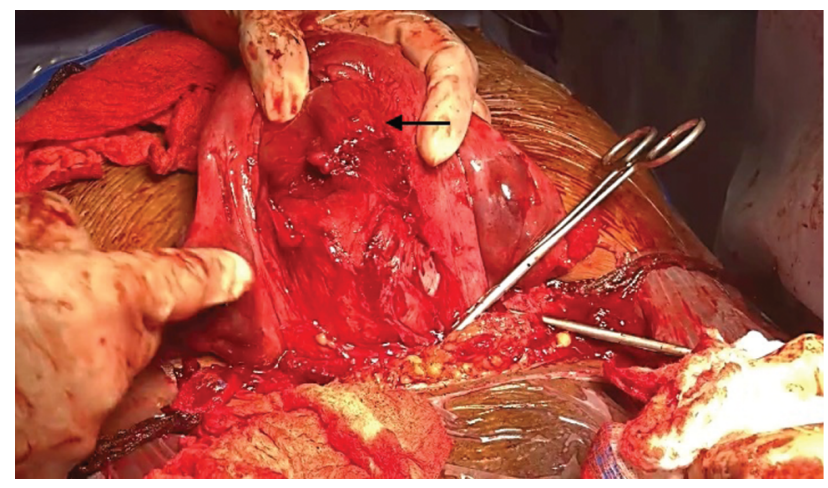

Figure 3: Transected adhesive band (arrow) after lysis of adhesions.

\section{Discussion}

Adhesion formation is a well-known complication of cesarean sections, with the risk increasing with each additional delivery [2]. Prior research on the neonatal complications in subsequent pregnancies has found an increased risk of malpresentation, laceration at time of delivery, and low Apgar scores due to prolonged intraoperative time to delivery [3]. Two concerns were raised at the time our patient was diagnosed with fetal head entrapment. The first was hindered future growth of the fetal head due to confinement within the lower uterine segment. Prior studies on other causes of cramped uterine environments, such as uterine didelphys, have reported increased risk for growth restriction, but only a single case report has described hindered growth as a result of fetal head entrapment [4], [5], [6]. Additionally, no literature has reported a similar location of entrapment within the lower uterine segment. In this case, the fetal head circumference was noted to be measuring less than the second percentile on ultrasound at 30 weeks' gestation, suggesting that the contracted space within the lower uterine segment can impede growth. Therefore, close monitoring with growth ultrasounds and potentially early delivery may be warranted in cases with similar findings.

The second concern raised at time of diagnosis was the potential for contractures of the fetal neck as a result of restricted movement. Fetal akinesia is a well-known cause of joint contractures and muscle abnormalities [7]. Fortunately, despite a fixed position of the fetal neck, there was no evidence of joint or muscle abnormalities after birth in this case.

The limitations of antenatal testing in predicting fetal status were also underscored with this patient. The objective of a BPP is to identify early fetal compromise, but the result needs to be interpreted carefully and in the context of the patient's condition. For example, in the case presented here, the low BPP score could be falsely low due to the ruptured membranes and presence of a uterine band restricting movement. In addition, the non-stress test, which is the most sensitive marker for predicting acute fetal hypoxia, was reactive. The limited utility of BPPs to assess fetal status was further supported by the infant's reassuring Apgar scores and normal cord blood gases at the time of delivery.

For this patient, a persistent inward bulge of the lower uterine segment was first identified during her routine anatomy scan. Given her history of two prior low transverse cesarean sections, the band of tissue was thought to be adhesive disease. The potential use of adjunct MRI was proposed in this case to better characterize the band of tissue. While ultrasound remains the gold standard obstetric screening modality, it has a sensitivity of only $43 \%$ for identifying adhesive disease [8]. Therefore, more often, awareness of these uterine bands does not occur until intraoperative identification. The adhesive band was diagnosed early in this case, which allowed the surgeon to anticipate potential intraoperative challenges, such as difficulty with delivery of the fetal head and damage to surrounding organs. The fibrous band within the underdeveloped lower uterine segment was intentionally avoided and a classical hysterotomy was done. As a result, the surgeon was able to navigate the uterine band to deliver the infant while avoiding unintentional bladder injury. Future research is needed on the utility of preoperative MRI to better characterize uterine adhesions to improve surgical planning.

With increasing rates of cesarean deliveries, adhesive disease will continue to pose many intraoperative challenges for the surgeon. There is very little literature on how these adhesions can affect the developing neonate. This case illustrates the implications of fetal head entrapment as a result of significant abdominal and lower uterine segment adhesions. When confinement occurs within the lower uterine segment, there may be increased risk of growth restriction of the fetal head and PPROM. In addition, this case highlighted the limitations of BPPs in predicting fetal status and the need for imaging modalities to better visualize adhesive disease for improved preoperative planning. 
Ethical approval: The research related to human use has complied with all the relevant national regulations, institutional policies and has been conducted in accordance with the tenets of the Helsinki Declaration, and it has been approved by the authors' Institutional Review Board or equivalent committee.

Author contributions: All the authors have accepted responsibility for the entire content of this submitted manuscript and approved submission.

Research funding: None declared.

Employment or leadership: None declared.

Honorarium: None declared.

Competing interests: The funding organization(s) played no role in the study design; in the collection, analysis, and interpretation of data; in the writing of the report; or in the decision to submit the report for publication.

\section{References}

[1] Clark EAS, Silver RM. Long-term maternal morbidity associated with repeat cesarean delivery. Am ] Obstet Gynec. 2011;205:S2-10.

[2] Tulandi T, Agdi M, Zarei A, Miner L, Sikirica V. Adhesion development and morbidity after repeat cesarean delivery. Am ] Obstet Gynec. 2009;201:56.e1-6.

[3] Deirdre JL. Adhesion and perioperative complications of repeat cesarean delivery. Am ] Obstet Cynec. 2011;205:S11-8.

[4] Venetis CA, Papadopoulos SP, Campo R, Gordts S, Tarlatzis BC, Grimbizis GF. Clinical implications of congenital uterine anomalies: a metaanalysis of comparative studies. Reprod Biomed Online. 2014;29:665-83.

[5] Roy-Lacrois M, Lambert I, Ouellet A. Fetal head entrapment between the maternal pelvic bone and a pelvic mass [abstract]. Ultrasound Obstet Gynecol. 2015;46:175.

[6] Deering SH, Heller], Winkel C, Landy H]. Intrauterine head entrapment of a second twin by a uterine synechia. Obstet Cynecol. 2003;102:693-5.

[7] Abraham R, Devi B. Fetal akinesia deformation sequence: a case report and review of literature. Int ] Reprod Contracept Obstet Cynecol. 2014;3:843-6.

[8] Lee M, Kim HS, Chung HH, Kim JW, Park NH, Song YS. Prediction of intra-abdominal adhesions using the visceral slide test: A prospective observational study. Eur] Obstet Gynecol Reprod Biol. 2017;213:22-5. 